\title{
CONSUMER SUPPORT OR DISAPPROVAL OF COMPANIES AS REVEALED BY SOCIAL BIG DATA
}

\author{
Jihyung Hong, Hyesun Hwang and Hyewon Lim \\ Sungkyunkwan University, South Korea \\ 25-2, Seonggyungwan-ro, Jongno-gu, Seoul, Republic of Korea
}

\begin{abstract}
Korean consumers have increasingly become aware of the significance of corporate social responsibility and unfairness issues of companies. Furthermore, as consumers are widely networked across the Internet, the reputation of companies can be rapidly disseminated online. The present study attempts to analyze online posts that are created by individual users of social media regarding consumers' different reactions to companies. Based on the result of related and emotional term analysis, we can identify the background to consumer support and disapproval of companies.
\end{abstract}

\section{KEYWORDS}

Consumer Support, Consumer Disapproval, Good Company, Boycott Company, Social Big Data

\section{INTRODUCTION}

Consumers have increasingly become aware of the significance of corporate social responsibility (Balabanis, 2013), leading to companies becoming concerned with benevolence. In South Korea, companies that exhibit benevolent and altruistic behaviors are termed "good companies" and they attract consumer support. Furthermore, as consumers become more aware of unfairness issues, corporate activities are being monitored by consumers (Shin \& Yoon, 2017). Finding unjustifiable activities can trigger consumer boycotts.

As consumers are widely networked across the Internet, the reputation of companies can be rapidly disseminated online. Social media in particular provide individual media to share one's own ideas with other people. In this context, research using text mining of social big data has become increasing popular, which is a very important data source (Bello-Orgaz, Jung, \& Camacho, 2016) for understanding consumer opinions. Consumers share information on both good and bad companies, which eventually affects corporate image and survival.

The purpose of this study is to investigate using text analysis the characteristics of companies that consumers support or disapproval. The result of this study will provide practical insights into understanding what stimulates consumer support or disapproval of companies, and how consumers feel about the companies.

\section{METHOD}

Online text created by consumers were collected using Trendup 4.0 that provides a platform to collect online text data from online news media and social media. The keywords to extract consumer opinions on good and bad companies were "good company" and "boycott company", respectively. We collected online text data that had been created between September 25, 2017 and September 25, 2018 on Twitter, Instagram, Blog, and other online communities.

We employed the term "good company" to generate the consumer support dataset because this term is much more familiar to Korean consumers, rather than "buycott", as a representative expression of consumer support including higher purchase intention and positive word-of-mouth (WOM). For the consumer disapproval dataset, "boycott company" was used as a keyword to extract consumer opinions on the disapproval and refusal to choose a particular companies' products or services. Based on the two datasets, the collected data were 
analyzed using text mining based on Korean natural language processing (KoNLP). To refine the data, stopwords were applied and extracted terms were classified into related and emotional terms according to the original meaning.

\section{RESULTS}

Firstly, the total number of texts about consumer support was 16,073, while the number of texts about consumer disapproval was 32,623 . This implies that negative reputation can be more widely and rapidly disseminated than positive reputation.

Secondly, the results of related term analysis showed that the major characteristics of companies attracting consumer support are "sponsor," "independence movement from Japanese imperialism," "disabled," "price," "social enterprise," "health," "wage," "environment," "philanthropy," and "donation." The terms are closely related to corporate social responsibility and indicate consumer awareness of a company's present and past activities. On the other hand, the major characteristics of consumer disapproval were "sexual discrimination," "misogyny," "male-dominated," "laborer," "corporate culture," "sexual assault," "new employee," "contract," "obedience," and "Zionism." As the "\#MeToo" movement has brought into prominence in South Korea, sexual discrimination was the most frequently mentioned term regarding consumer disapproval. Also the result shows that consumers are aware of organizational structure through focusing on lower level staff members such as new employees and laborers. This indicates that consumers are concerned about moral issues rather than product deficiency or typically unfair market practices.

Thirdly, the positive terms related to consumer support were "good," "cheap," "various," "pretty," "necessary," "new," "healthy," "honest," "reliable," and "safe." This indicates that consumers evaluate company performance based on criteria such as price or quality of products or services, in determining good companies. On the other hand, the negative terms related to consumer disapproval were "serious," "thoughtless," "insulted," "harmful," "corrupt," "difficult," "disgusted," "dirty," "blame," and "irrational." These results show that consumer disapproval is not only related to a company's poor performance but also related to immoral or unethical acts.

Table 1. Results of related term analysis

\begin{tabular}{ccccc}
\hline & \multicolumn{2}{c}{ Consumer support of companies } & \multicolumn{2}{c}{ Consumer disapproval of companies } \\
\cline { 2 - 5 } Rank & Related term & Frequency & Related term & Frequency \\
\hline 1 & Sponsor & 5654 & Sexual discrimination & 13825 \\
2 & Independence movement & 3857 & Misogyny & 6812 \\
3 & Disabled & 2662 & Male-dominated & 3537 \\
4 & Price & 2618 & Laborer & 3146 \\
5 & Social enterprise & 2019 & Corporate culture & 3047 \\
6 & Health & Sexual assault & 2884 \\
7 & Wage & 1575 & New employee & 2852 \\
8 & Environment & 1184 & Contract & 2843 \\
9 & Philanthropy & 1151 & Obedience & 2840 \\
10 & Donation & 1134 & Zionism & 2066 \\
11 & Protection & 1105 & Racial discrimination & 2066 \\
12 & Overseas & 1085 & Interview & 1835 \\
13 & Welfare & 1033 & Litigation & 1405 \\
14 & Nature & Coercion & 1168 \\
15 & Children & 971 & Advertisement & 1043 \\
16 & Voluntary & 968 & Feminist & 765 \\
17 & Safety & 965 & Connivance & 603 \\
18 & Honesty & Murder & 478 \\
19 & One-man company & 895 & Unfair dismissal & 456 \\
20 & Eco-friendly & Exploitation & 448 \\
\hline
\end{tabular}


Table 2. Results of emotional term analysis

\begin{tabular}{ccccc}
\hline & \multicolumn{2}{c}{ Consumer support of companies } & \multicolumn{2}{c}{ Consumer disapproval of companies } \\
\cline { 2 - 5 } Rank & Positive term & Frequency & Negative term & Frequency \\
\hline 1 & Good & 4295 & Serious & 2250 \\
2 & Cheap & 4056 & Thoughtless & 1823 \\
3 & Various & 1889 & Insulted & 1772 \\
4 & Pretty & 1803 & Harmful & 597 \\
5 & Necessary & 1603 & Corrupt & 340 \\
6 & New & 926 & Difficult & 266 \\
7 & Healthy & 909 & Disgusted & 220 \\
8 & Honest & 869 & Dirty & 207 \\
9 & Reliable & 860 & Blame & 164 \\
10 & Safe & 809 & Fearful & 144 \\
11 & Highly recommended & 793 & Unfair & 138 \\
12 & Quickly & 763 & Uncomfortable & 98 \\
13 & Benevolent & 762 & Angry & 90 \\
14 & Thankful & 744 & Grudge & 87 \\
15 & Soft & 672 & Vain & 78 \\
16 & Excellent & 625 & Odd & 77 \\
17 & Easily & 615 & Wrong & 77 \\
18 & Happy & 611 & Rensitive & 75 \\
19 & Beloved & 606 & 71 \\
20 & Famous & 599 & 71 \\
\hline
\end{tabular}

\section{CONCLUSION}

The current study explored the background to consumer support and disapproval of companies by analyzing consumers' words posted online. In particular, broadening themes for boycotting can be observed. From the failure of business or legality issues to immoral or organizational culture issues, consumers oppose unfairness in society. Based on the result of this study, we can identify the background to consumer support and disapproval of companies. This can provide a guide used to direct companies towards sustainability.

\section{REFERENCES}

Balabanis, G., 2013. Surrogate Boycotts against Multinational Corporations: Consumers' Choice of Boycott Targets. British Journal of Management, Vo1. 24, No. 4, pp. 515-531.

Bello-Orgaz, G. et al, 2016. Social Big Data: Recent Achievements and New Challenges. Information Fusion, Vol. 28, pp. 45-59.

Shin, S. Y. and Yoon, S. W., 2017. A Target Company's Offending Action Reduced Negative Emotions and Consumer Boycott Decision Making. Journal of Consumption Culture, Vol. 20, No. 4, pp. 93-116. 